\title{
AMERICAN BOARD OF MEDICAL GENETICS DIPLOMATES
}

The American Board of Medical Genetics is pleased to announce the diplomates certified in 1999:

\author{
Clinical Geneticists (89) \\ Ahmad, Aỵesha \\ Al-Swaid, Abdulrahman Faiz \\ Alsayed, Moeenaldeen D. A. \\ Arch, Ellen N. \\ Babovic-Vuksanovic, Dusica \\ Bamshad, Michael J. \\ Barabas, Ronald E. \\ Bejiani, Bassem A. \\ Blakley, Patricia $M$. \\ Bodamer, Olaf $A$. \\ Boerkoel, Cornelius F. \\ Boyadiiev, Simeon A. \\ Brooks, David G. \\ Brown, Chester W. \\ Chen, Emily \\ Chong, Karen L. \\ Del Campo, Miguel \\ Eddleman, Keith A. \\ Enns, Gregory M. \\ Everman, David B. \\ Eyaid, Wafaa \\ Feldman, Baruch \\ Ferreira, Jose Carolos P. B. \\ Gambello, Michael J. \\ Gomez, Kevin J. \\ Gripp, Karen W. \\ Gropman, Andrea L. \\ Gunay-Aygun, Meral \\ Gupta, Gopal K. \\ Hahn, Si H. \\ Hannibal, Mark C. \\ Heilstedt, Heidi A. \\ Henderson, Leon B. \\ Hershberg, Robert M. \\ Hisama, Fuki M. \\ Huang, Taosheng \\ Ierardi-Curto, Lynne A. \\ Iglesias, Alejandro D. \\ Johnson, Dennis R. \\ Karimi, Ardeshir \\ Kostiner, Dana R. \\ LaSpada, Albert R. \\ Lazebnik, Noam \\ Lee, Brendan Hon Leung \\ Levy, Howard $P$. \\ Limwongse, Chanin \\ Linck, Leesa M. \\ Lipkin, Steven M \\ Matern, Dietrich \\ McCandless, Shawn E. \\ McClellan, Michael W. \\ McFarland, Melinda B. \\ Milunsky, Jeff $M$. \\ Ming, Jeffrey E. \\ Musci, Thomas J. \\ Nathanson, Katherine \\ Nunes, Mark E. \\ Parisi, Melissa A. \\ Patel, Millan S.
}

Plump, Andrew S.

Ponkey, Susan E.

Repetto, Gabriela M.

Roberts, Jaclyn L.

Roberts, Michele

Rose, Verna M.

Saitta, Sulagna Chakraborty

Santolaya-Forgas, Joaquin

Scaglia, Fernando

Schowalter, David B.

Shotelersuk, Vorasuk

Siegel-Bartelt, Jacqueline

Sigmundsson, Jon

Smith, Rosemarie

Stewart, Theresa L.

Stockton, David W.

Storm, Andrea

Summar, Marshall L.

Sutton, Vernon R.

Swoboda, Kathryn I.

Tsai, Chun-Hui

Unger, Sheila L.

Vilain, Eric J. N.

Waggoner, Darrel J.

Walsh, Laurence E.

Wasserstein, Melissa P

Wattanasirichaigoon, Duangrurd

Wheeler, Patricia G.

Yaron, Yuval

Zilberstein, Moshe E.

Clinical Biochemical Geneticists (21)

Al-Aqeel, Aida I.

Alsayed, Moeenaldeen D. A.

Arch, Ellen $\mathrm{M}$.

Elsea, Sarah H

Enns, Gregory M.

Finegold, David N.

Gibson, Kenneth $M$.

Hahn, Si H.

Iglesias, Alejandro D

Koeberl, Dwight D.

Kronn, David F.

Lee, Brendan Hon Leung

Linck, Leesa $M$.

Longo, Nicola

Qu, Yong

Raff, Michael L.

Shotelersuk, Vorasuk

Summar, Marshall L.

Vilain, Eric I. N

Wasserstein, Melissa P.

Yu, Chunli

Clinical Cytogeneticists (31)

Adhvaryu, Siddharth G.

Barnoski, Barry L.

Berend, Sue Ann

Berry, Rebecca S.

Blough, Ruthann Ida

\author{
Cargile, Colyn B. \\ Carlson, Katrin M. \\ Crawford, Eric \\ Dong, Liang \\ Drumheller, Timothy C. \\ El-Naggar, Mohamed M \\ Gastier-Foster, Julie M. \\ Giersch, Anne B. \\ Hibbard, Michele $\mathrm{K}$. \\ Knops, Judith F. \\ Koty, Patrick \\ Kumar, Arun \\ Lese, Christa M. \\ Li, Chumei \\ Ligon, Azra $\mathrm{H}$. \\ Ning, Yi \\ Praphanphoj, Verayuth \\ Pringle, $M$. Jodeane \\ Stewart, Sophia D. \\ Thurston, Virginia \\ Tsuchiya, Karen D. \\ Winters, Jennifer L. \\ Xiao, Sheng \\ Xu, Jie \\ Yu, Jingwei \\ Zhang, Xiao-Xiang
}

Clinical Molecular Geneticists (57)

Aksentijevich, Iovona

Ashton-Prolla, Patricia

Babovic-Vuksanovic, Dusica

Bali, Deeksha

Bao, Liming

Bellissimo, Daniel B.

Brock, Jo-Ann K.

Chen, Emily

Chen, Tian-Jian

Chong, Samuel S.

Coberly, Suzanne $k$.

Crawford, Eric

Desnick, Robert J.

El-Naggar, Mohamed M.

Fan, Fan

Fodor, Flora $\mathrm{H}$.

Ganguly, Arupa

Gastier-Foster, Julie M.

Gripp, Karen W.

Gunaratne, Preethi $\mathrm{H}$.

Huang, Taosheng

Ito, Masamichi

Iyer, Ramaswamy K.

Kolosha, Vladimir O.

LaSpada, Albert R.

Lee-Kirsch, Min A. E.

Li, Lei

Li, Peining

Longshore, John W.

Lubin, Ira M.

McAndrew, Patricia E.

McDowell, Geraldine A. 
Milunsky, Jeff M.

Monaghan, Kristin G.

Palmer, Susan E.

Paperna, Tamar Y.

Qi, Ming

Qu, Yong

Qumsiyeh, Mazin

Reinartz, John J.

Rennert, Hanna

Ring, Huijun Z.
Rohlfs, Elizabeth M.

Schrijver, Iris

Sirko-Osadsa, Deborah

Skarpidi, Evangelia

Stenzel, Timothy

Sun, Weimin

Telatar, Milhan

Toland, Amanda Ewart

Tsuchiya, Karen D.

Venkatraj, Vijayanagaram S.
Vo, Trieu D.

Vulpe, Christopher D.

Wattanasirichaigoon, Duangrurd

Wiggs, Janey L.

Yano, Shoji

PhD Medical Geneticists (3)

Monaghan, Kristin $\mathrm{G}$.

Richard, Gabriele

Toland, Amanda Ewart

\section{AMERICAN BOARD OF GENETIC COUNSELING}

The American Board of Genetic Counseling is pleased to announce the diplomates certified in 1999:

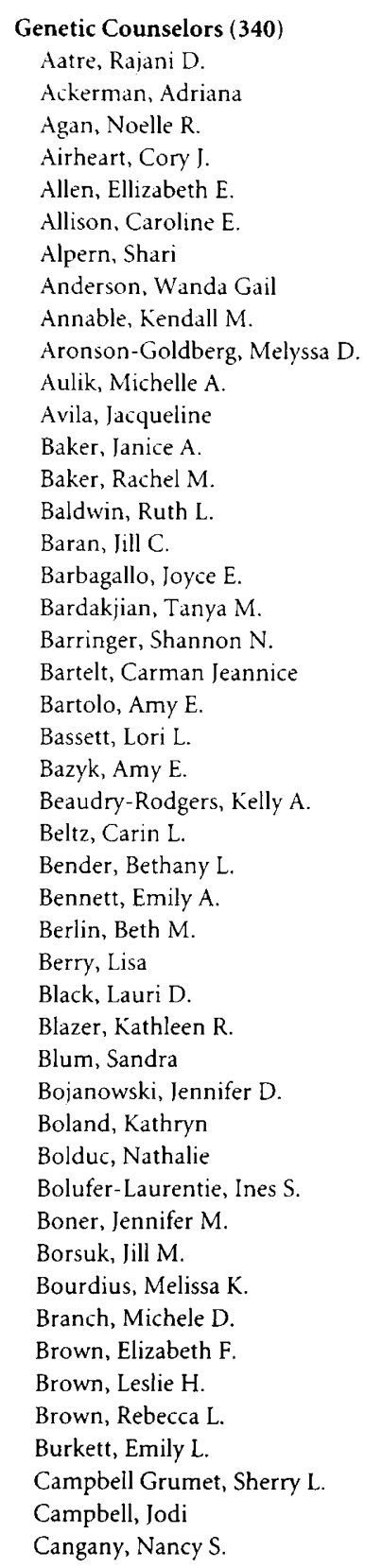

Carmody, Jennifer L.

Causey, Tahnee N.

Cavanagh, Trudy A.

Celle, Livija

Challinor, Regan M.

Chamberlain, Kristine B.

Chandy, Nandini M.

Chen, Elizabeth A.

Chen, Laurie L.

Chen, Margaret A.

Cheney, Susan M.

Chijiwa, Chieko

Chittenden, Anuradha B.

Chow, Norma

Christian, Susan M.

Clouser, Pamela J.

Clyman, Jonathan C.

Connerton-Moyer, Kelly J.

Conrad, Margaret G.

Conway, Laura J.

Cooper, Heather A.

Correia, Lori Ann

Coryell, R. Jason

Cowley, Catherine S.

Crowley, Amy E.

Crowley, Kerry E.

Culler, Duane D.

Culp, Kristin B.

Curell-Langbo, Carrie J.

Daley, Katherine E.

Danziger, Kari L.

Davidson, Suzanne R.

Decker, Amy B.

Del Vecchio, Maria A.

Delgado, Dalisay M.

Demarco, Kristin A.

Dent, Karin M.

Deprince, Kristin M.

Deriso, Natalie L.

Devers, Patricia L.

Diaz-Barrios, Vivien

Dickinson, Susan L. J.

Diekmann, Dagmar B.

Dietrich S. Katherine

DiGiulio, Kathleen A.

Dircks, Anita M.

Donahoo, Kristen L.

Dorman, Heather
Dougan, Shelley D.

Drain, Wendy M.

Dunstone, Robin L.

Dupuis, Lucie

Duryea, Melinda F.

Eilers, Margaret L.

Einy, Reviva

Estabrooks, Susan E.

Facher, Jennifer J.

Farmer, Jennifer M.

Fedosoff, Kimberly M.

Feiger, Jennie W.

Ferguson, Heather L.

Ferner, Dena

Fleming, Dusty L.

Flore, Leigh Anne

Follmer, Jaime E.

Forrester, Shawnia R.

Fowler, Eric S.

Freehauf, Cynthia L.

Fuchs, Andrea E.

Gaebe Schulz, Tracey L.

Gall, Kimberly A.

Garza, Judy

Ghate, Sumedha

Gibbons, Clare A.

Graf, Michael D.

Grant, Deniera S.

Graveline, Johanna

Graw, Sharon L.

Griffin, Anne C.

Grinshpun-Cohen, Julia

Grund, Jaime E.

Guimond, Colleen H.

Gustin. Joy A.

Guy Malloy, Athena C.

Habecker-Green, Julia G.

Hall, Donita I.

Hammer, Gail

Hanna, Danielle E.

Hanson, Kristine $\mathrm{K}$.

Hartley, Nicole $M$.

Haymowicz, Heather L.

Hedrick, Julie F.

Heer, Nicole B.

Helin, Kara L.

Hewson, A. Stacy

Hirji, Riyana 
Horess, Karen B.

Hogan, Molly L.

Homer, Jeanne P.

Homeyer, Margaret $M$.

Hoodtar, Elizabeth

Horsma, Jennifer L.

Hoyle, Cynthia L.

Hubbard, Gail L.

Huggins, Marlene I.

Hulait, Gurdip K.

Hulsebos, Yvonne L.

Hunter, Jennifer A.

Ingram, Susan M.

Isaac, Nisha

Ishael, Holly A.

Jackson, Judith A

Jarrett, Kristine L.

Johnson, Kimberly S.

Johnson, Nancy \$.

Johnson, Sara M.

Jordan, Lindsay E.

Kamen, Heather $T$.

Kasprzak, Lidia Z.

Kearney, Elizabeth $M$

Keller, Kory L.

Kennedy, Shelley

Kerper, Peggy S.

Kim, Katherine $H$.

Kirsch, Jodi P.

Koehn, David C.

Kohan, Naghmeh Melody M.

Kohlmann, Wendy K.

Krajewski, Karen M.

Kramer, Nancy A.

Kramer, Stephanie B.

Krasner, Meagan R.

Krebs, Valerie $\mathrm{R}$.

Kruisselbrink, Teresa M.

Ku, Lisa

Kubas, Cheryl

Kushner, Jessica D.

Lasarsky, Nicole B.

Lee, Heather M.

Leight, Ivy S.

Lesh, J. Shonee

Lewis, Dorothy S.

Lilley, Margaret A.

Lintner, M. Denise

Loebl, Charli

Loik, Valerie I.

Lorentz, Cindy Pham

Lucente, Diane E.

Lundberg, Jill $\mathrm{K}$.

Lynch, Katherine A.

Malabed, Katherine L.

Mandell, Jessica

Marcy, Jennifer

Masciangelo, Christina M.

Masser-Frye, Diane S.

Matika, Gretchen L.

McBride, Robin E.

McDermott, Deborah A.

McInerney, Aideen M.

McLoughlin, Reagan

Melchert, Lael E.

Mensching, Lisa C.
Merkel, Heather A.

Meyer, Allyson N.

Michels, Ann L.

Miller, Mary H.

Miller, Stacy A.

Mills, Carolyn M.

Mills, Linda $\mathrm{K}$.

Mills, Patti L.

Moore, Christine R.

Moore, Michelle M.

Morley, Gina M.

Mueller, Shelley A.

Murdoch, Julianne

Murray, Michele S.

Nagel, Heidi L.

Nash, Katherine B.

Naumer, Anne C.

Naylor, Joan M.

Neil, Jennifer E.

Neufeld-Kaiser, Whitney A.

Nichols, Jerilynn R.

Niendorf, Kristin B.

Niewiadomski, Laura A.

Noll, Claire L.

Norris, Virginia W.

O Neill, Suzanne M.

O Sullivan, Corrine K.

Oelrich, Mary K

Ohle, Carolyn

Ostrovsky, Ariela D.

Owen, Stacy C.

Ozaki, Jennifer $\mathrm{H}$.

Padellford, Tressa L.

Page, Kristi L.

Page, Patricia Z.

Palmer, Maureen M.

Panabaker, Karen L.

Paras, Andrea

Patterson, Melissa A.

Paul, Anne E.

Pearlman, Charlene L.

Peasley, Ericka B.

Peay, Holly Landrum

Petrucelli, Nancie

Pierce, Heather Hanson

Pietryga, Marguerite

Pinto, Mark D.

Pollin, Toni I.

Poss, Alexis $\mathrm{F}$.

Pouncey, Jill

Powell, Erin M.

Prensky, Lawrence M.

Queneau, Michelle J.

Quercia, Nada L.

Quillin, John M

Randall-Pinto, Susan

Ravan, Sarah A.

Raymond, Deborah

Reed, Lori

Rimar, Elena

Rittenhouse, Kimberley R.

Roggenbuck, Jennifer A.

Rosa, Deborah A.

Rosenthal, Eric T.

Rossello, Kathleen C.

Roth, Margaret K.
Rothenmund, Heidi

Rubin, Karol R.

Russell, Karen L.

Sawyer, Jill C.

Sawyer, Julie

Schain, Katherine L.

Schmidt, Diane L.

Schmidt, Stacy L.

Schrock-Kelley, Suzanne

Schwandt, Kirstin J.

Shane, Heather

Shannon, Kristen E.

Shaw, Martha E.

Shrigley, Rebecca J.

Siegel, Jennifer R.

Singletary, Claire N.

Smith, Ursula A.

Smyth, Kathleen A.

Snow, Stephanie R.

Speare, Virginia J.

Stebner, Karen E.

Stein, Laura L.

Steinwachs, Ellen F.

Stenzel, Anabel M.

Stephan, Yolanda M.

Stettner, Amy R.

Stone, Kristyne $\mathbf{M}$.

Studinski, April L.

Sullivan, Jennifer A.

Sundaram, Usha S.

Swanson, Karen J.

Swanson, Korin C.

Tatlock, Amanda I.

Teicher, Jennifer D.

Terry, Lori A.

Thompson, Elizabeth A.

Toburen, Amy L.

Trant, Melissa

Tripi, Rachael K.

Tucker, Aimee J.

Tucker, Bethany D.

Tuttle, Lisa C.

Ungerleider, Sharon B.

Vargo, Diane S.

Verbrugge, Jennifer L.

Vignovich, Joan

Vikstrom, Karen F.

Vimb, Aino

Vininsky, Rochelle

Walsh, Amanda J.

Walter, Aimee L.

Wans, Kathleen C.

Weaver, Amybeth $\mathrm{M}$.

Weisiger, Kara L.

Welch, Karlla K.

Wells, Deborah F.

Williamson Catania, Jennifer

Willis, Barbara F.

Wong, Stacey A.

Wright, William W.

Yamada, Laura

Yashar, Beverly M.

Ybarra, Andrea A.

Yonago, Jill S.

Ziebarth, Ingrid $\mathrm{N}$. 